\title{
THE LCR OF WIRELESS MACRODIVERSITY SSC RECEIVER IN THE PRESENCE OF GAMMA SHADOWED KAPPA-MU FADING
}

\section{Časlav Stefanović ${ }^{1}$, Danijel Đošić ${ }^{2 *}$}

${ }^{1}$ Faculty of Natural Sciences and Mathematics, University of Priština, Kosovska Mitrovica, Serbia.

\section{ABSTRACT}

Wireless mobile macrodiversity (MaD) radio system with switch and stay (SSC) receiver and two microdiversity (MiD) selection combining (SC) branches operating over Gamma shadowed Kappa-Mu $(\boldsymbol{k}-\boldsymbol{\mu})$ multipath fading environment is considered. Novel, one-folded integral expression for average level crossing rate (LCR) of MaD SSC receiver output signal envelope is obtained. Numerical results of the proposed model are presented and discussed in relation to the system model parameters.

Key words: LCR, MaD, MiD, multipath, shadowing.

\section{INTRODUCTION}

Macrodiversity (MaD) system consisting of $\mathrm{MaD}$ receiver and two or more microdiversity (MiD) branches is often proposed in the literature ((Shankar, 2009), (Mukherjee \& Avidor, 2003), (Basnayaka et al., 2013).

Moreover, multipath fading can be mitigated by $\mathrm{MiD}$ receivers at single $\mathrm{BSs}$ (base stations) while shadowing can be mitigated by $\mathrm{MaD}$ receiver combining signals from two or more BSs. It is important to note that multipath fading is caused by physical phenomena such as reflection, refraction and scattering of radio waves while shadowing is caused by obstacles between transmitter and receiver.

$\mathrm{MaD}$ switch and stay (SSC) system with MiD selection combining (SC) as well as MaD SC system with MiD maximal ratio combining (MRC) or SC can be efficiently applied in composite fading environment to improve performances of wireless communication system as shown in((Stefanović, 2015), (Djosic et al., 2016), (Stefanović et al., 2016), (Panić et al., 2011), (Stamenović et al., 2014)).

The Kappa-Mu $(\kappa-\mu)$ distribution describes signal envelope variation in line-of-sight (LoS) multipath fading channels when signals propagate in the environment with two or more clusters. Parameter $k$ is known as Rician factor and can be calculated as the ratio of dominant components power and scattering components power while parameter $\mu$ is related to the number of clusters in the propagation environment. Moreover, kappa-mu is general distribution, which means that for different values of $\kappa$ and $\mu$, Rayleigh, Rice and Nakagami- $m$ distributions can be derived. Moreover, this distribution fits well with experimental data and is often applied in multipath environment ((Yacoub, 2007), (Paris, 2014), (Stefanović et al., 2015)).

Log-normal distribution and Gamma distribution are used for the purpose of describing shadowing in wireless communication channel.

In this paper, MaD system has been modeled with switch and stay (SSC) receiver at macro level and two dual branch selection combining (SC) at micro level, since its relatively low implementation complexity. Morover, SC and SSC are often considered diversity techniques ((Zhang et al., 2014), (Milic et al., 2016), (Stefanovic et al., 2013), (Zhao et al., 2015)). Gamma distribution is proposed, with the tendency of obtaining closed form solutions due to its mathematical tractability. Moreover, the average level crossing rate (LCR) of $\mathrm{MaD} \mathrm{SSC}$ receiver output signal envelope in the presence of Gamma shadowed $k-\mu$ multipath fading is efficiently calculated in the form of the sum of one-folded integral. Numerical results are presented graphically and the effect of Rician factor, multipath fading severity parameter, shadowing severity parameter, correlation coefficient and threshold of SSC on the proposed MaD model are examined. 


\section{SYSTEM MODEL}

The MaD system with two MiD SC parts under the influence of Gamma shadowed $k-\mu$ multipath fading as shown on Fig. 1. is considered. Operation is the following: when signal envelope average power at the inputs of the first $\mathrm{SC}, \Omega_{1}$ is higher than a specified threshold, $\Omega_{T}$, MaD SSC selects the first SC to process the signal to the receiver. Contrary, when $\Omega_{1}$ is lower than $\Omega_{T}$, SSC selects the second MiD SC to provide the signal path. At the micro level, MiD SC selects the branch with the highest signal envelope power to provide the signal to receiver.

Input signal envelopes for the first MiD path are denoted with $y_{11}$ and $y_{12}$, while the second MiD input signal envelopes are denoted with $y_{21}$ and $y_{22}$. Further, the output signal envelopes of the first and second MiD combiners are $y_{1}$ and $y_{2}$, respectively, while signal envelope at the output of $\mathrm{MaD} \mathrm{SSC}$ is denoted with $y$.

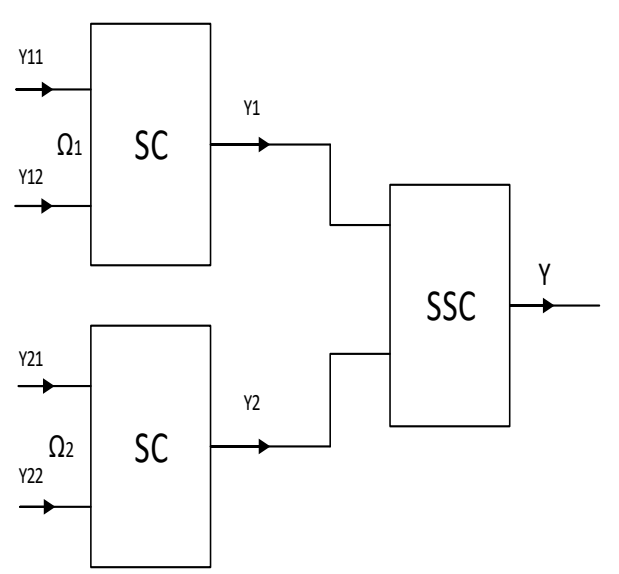

Fig. 1 The MaD system with MaD SSC structure and two MiD SC parts.

Probability density function (PDF) of $\kappa-\mu$ signal envelope at the inputs of the first SC are respectively (Panic et al., 2013):

$$
\begin{aligned}
p_{y_{1 i}}\left(y_{1 i}\right)= & \frac{2 \mu(k+1)^{\frac{\mu+1}{2}}}{k^{\frac{\mu-1}{2}} e^{k \mu} \Omega_{1}{ }^{\frac{\mu+1}{2}}} y_{1 i}{ }^{\mu} I_{\mu-1}\left(2 \mu \sqrt{\frac{k(k+1)}{\Omega_{1}}}\right) . \\
& e^{-\frac{\mu(k+1)}{\Omega_{1}} y_{1 i}{ }^{2}}, \quad i=1,2 .
\end{aligned}
$$

where, $\kappa$ is Rice factor, $\mu$ is fading severity factor, $\Omega_{1}$ is related to the local mean power of $y_{1 i}$ and $I_{v}(\cdot)$ is the modified Bessel function of the first kind and order $v$ (Stefanovic et al., 2013). The $I_{v}(\cdot)$ can be transformed by utilization (Gradshteyn \& Ryzhik,
2000), so that the CDF expression of $k-\mu$ signal envelope at the inputs of the first SC can be expressed as (Stüber, 1996):

$$
\begin{aligned}
& F_{y_{1 i}}\left(y_{1 i}\right)=\int_{0}^{y_{1 i}} p_{y_{1 i}}(t) d t=\frac{2 \mu(k+1)^{\frac{\mu+1}{2}}}{k^{\frac{\mu-1}{2}} e^{k \mu} \Omega_{1}^{\frac{\mu+1}{2}}} \\
& \cdot \sum_{j=1}^{\infty}\left(\mu \sqrt{\frac{k(k+1)}{\Omega_{1}}}\right)^{2 j+\mu-1} \frac{1}{2}\left(\frac{\Omega_{1}}{\mu(k+1)}\right)^{j+\mu} . \\
& \cdot \frac{1}{j ! \Gamma(j+\mu)} \gamma\left(j+\mu, \frac{\mu(k+1)}{\Omega_{1}} y_{1 i}{ }^{2}\right) .
\end{aligned}
$$

where $\Omega_{1}$ is signal envelope average power at input of first micro-diversity SC and $\gamma(m, x)$ is incomplete Gamma function. CDF of the first SC output signal envelope is:

$$
\begin{aligned}
& F_{y_{1}}\left(y_{1}\right)=F_{y_{11}}\left(y_{1}\right) F_{y_{12}}\left(y_{1}\right)=\left(\frac{2 \mu(k+1)^{\frac{\mu+1}{2}}}{k^{\frac{\mu-1}{2}} e^{k \mu} \Omega_{1}^{\frac{\mu+1}{2}}}\right)^{2} . \\
& \cdot \sum_{i_{1}=1}^{\infty}\left(\mu \sqrt{\frac{k(k+1)}{\Omega_{1}}}\right)^{2 i_{1}+\mu-1} \frac{1}{2}\left(\frac{\Omega_{1}}{\mu(k+1)}\right)^{i_{1}+\mu} \frac{1}{i_{1} ! \Gamma\left(i_{1}+\mu\right)} . \\
& \cdot \gamma\left(i_{1}+\mu, \frac{\mu(k+1)}{\Omega_{1}} y_{1}^{2}\right) \sum_{i_{2}=1}^{\infty}\left(\mu \sqrt{\frac{k(k+1)}{\Omega_{1}}}\right)^{2 i_{2}+\mu-1} \cdot \\
& \cdot \frac{1}{2}\left(\frac{\Omega_{1}}{\mu(k+1)}\right)^{i_{2}+\mu} \frac{1}{i_{2} ! \Gamma\left(i_{2}+\mu\right)} \gamma\left(i_{2}+\mu, \frac{\mu(k+1)}{\Omega_{1}} y_{1}^{2}\right) .
\end{aligned}
$$

Similarly, CDF of SC output $k-\mu$ random variable $y_{2}$ is:

$$
\begin{aligned}
& F_{y_{2}}\left(y_{2}\right)=F_{y_{21}}\left(y_{2}\right) F_{y_{22}}\left(y_{2}\right)=\left(\frac{2 \mu(k+1)^{\frac{\mu+1}{2}}}{k^{\frac{\mu-1}{2}} e^{k \mu} \Omega_{2}^{\frac{\mu+1}{2}}}\right)^{2} . \\
& \cdot \sum_{i_{1}=1}^{\infty}\left(\mu \sqrt{\frac{k(k+1)}{\Omega_{2}}}\right)^{2 i_{1}+\mu-1} \times \frac{1}{2}\left(\frac{\Omega_{2}}{\mu(k+1)}\right)^{i_{1}+\mu} \frac{1}{i_{1} ! \Gamma\left(i_{1}+\mu\right)} \cdot \\
& \cdot \gamma\left(i_{1}+\mu, \frac{\mu(k+1)}{\Omega_{2}} y_{2}^{2}\right) \sum_{i_{2}=1}^{\infty}\left(\mu \sqrt{\frac{k(k+1)}{\Omega_{2}}}\right)^{2 i_{2}+\mu-1} \cdot \\
& \cdot \frac{1}{2}\left(\frac{\Omega_{2}}{\mu(k+1)}\right)^{i_{2}+\mu} \frac{1}{i_{2} ! \Gamma\left(i_{2}+\mu\right)} \gamma\left(i_{2}+\mu, \frac{\mu(k+1)}{\Omega_{2}} y_{2}^{2}\right) .
\end{aligned}
$$

Signal envelope average power at inputs of two MiD SC structures, $\Omega_{1}$ and $\Omega_{2}$ are Gamma distributed ((Xekalaki et al, 2003), (Yue et al., 2001)): 


$$
\begin{aligned}
& p_{\Omega_{1} \Omega_{2}}\left(\Omega_{1} \Omega_{2}\right)=\frac{\left(\Omega_{1} \Omega_{2}\right)^{\frac{c-1}{2}}}{\Gamma(c)\left(1-\rho^{2}\right) \rho^{c-1} \Omega_{0}^{c+1}} e^{-\frac{\Omega_{1}+\Omega_{2}}{\Omega_{0}\left(1-\rho^{2}\right)}} . \\
& \cdot I_{c-1}\left(\frac{2 \rho}{\Omega_{0}\left(1-\rho^{2}\right)} \Omega_{1} \Omega_{2}{ }^{\frac{1}{2}}\right)= \\
& =\frac{1}{\Gamma(c)\left(1-\rho^{2}\right) \rho^{c-1} \Omega_{0}^{c+1}} \sum_{i=0}^{\infty}\left(\frac{\rho}{\Omega_{0}\left(1-\rho^{2}\right)}\right)^{2 i+c-1} . \\
& \cdot \frac{1}{i ! \Gamma(i+c)} \Omega_{1}^{i+c-1} \Omega_{2}^{i+c-1} e^{-\frac{\Omega_{1}+\Omega_{2}}{\Omega_{0}\left(1-\rho^{2}\right)}} .
\end{aligned}
$$

where $\rho$ is correlation coefficient of the shadowing process, $c$ is shadowing parameter and $\Omega_{0}$ is mean value of $\Omega_{1}$ or $\Omega_{2}$.

\section{LCR OF MAD SSC RECEIVER OUTPUT SIGNAL}

LCR of Kappa-Mu random variable $y_{1 i}$ is (Panic et al., 2003):

$$
\begin{aligned}
N_{y_{1 i}} & =\int_{0}^{\infty} \dot{y} p_{y_{1 i} \dot{y}_{1 i}}\left(y_{1 i} \dot{y}_{1 i}\right) d \dot{y}_{1 i}= \\
& =\frac{\sqrt{2 \pi} f_{m} 2 \mu^{\frac{1}{2}}(k+1)^{\frac{\mu}{2}}}{k^{\frac{\mu-1}{2}} e^{k \mu} \Omega_{1}^{\frac{\mu}{2}}} \sum_{i_{i}=1}^{\infty}\left(\mu \sqrt{\frac{k(k+1)}{\Omega_{1}}}\right)^{2 i_{1}+\mu-1} . \\
& \frac{1}{i_{1} ! \Gamma\left(i_{1}+\mu\right)} y_{1 i}^{2 i_{1}+2 \mu-1} e^{-\frac{\mu(k+1)}{\Omega_{1}} y_{1 i}{ }^{2}}, i=1,2 .
\end{aligned}
$$

where $p_{y_{i} \dot{y}_{1 i}} y_{1 i} \dot{y}_{1 i}$ is joint probability density function (JPDF) of $y_{1 i}$ and its derivative, $f_{m}$ is maximal Doppler frequency.

Similarly, LCR of random variable $y_{2 i}$ is:

$$
\begin{gathered}
N_{y_{2 i}}=\frac{\sqrt{2 \pi} f_{m} 2 \mu^{\frac{1}{2}}(k+1)^{\frac{\mu}{2}}}{k^{\frac{\mu-1}{2}} e^{k \mu} \Omega_{2}^{\frac{\mu}{2}}} \sum_{i_{1}=1}^{\infty}\left(\mu \sqrt{\frac{k(k+1)}{\Omega_{2}}}\right)^{2 i_{1}+\mu-1} . \\
\cdot \frac{1}{i_{1} ! \Gamma\left(i_{1}+\mu\right)} y_{2 i}{ }^{2 i_{1}+2 \mu-1} e^{-\frac{\mu(k+1)}{\Omega_{2}} y_{2 i}{ }^{2}}, i=1,2 .
\end{gathered}
$$

Since the fading is identical and independent, JPDF of the MiD SC output signal envelope $y_{1}$ and its first derivative is:

$$
\begin{aligned}
p_{y_{1} \dot{y}_{1}}\left(y_{1} \dot{y}_{1}\right) & =p_{y_{11} \dot{y}_{11}}\left(y_{1} \dot{y}_{1}\right) F_{y_{12}}+p_{y_{12} \dot{y}_{12}}\left(y_{1} \dot{y}_{1}\right) F_{y_{11}}\left(y_{1}\right)= \\
& =2 p_{y_{1} \dot{y}_{11}}\left(y_{1} \dot{y}_{1}\right) F_{y_{12}}\left(y_{1}\right) .
\end{aligned}
$$

The LCR of the first MiD SC output signal envelope $y_{1}$ is:

$$
\begin{aligned}
& N_{y_{1}}=\int_{0}^{\infty} \dot{y}_{1} p_{y_{1} \dot{y}_{1}}\left(y_{1} \dot{y}_{1}\right) d \dot{y}_{1}=2 F_{y_{12}}\left(y_{1}\right) N_{y_{11}}= \\
& =\frac{2 \sqrt{2 \pi} f_{m} \mu^{\frac{1}{2}}(k+1)^{\frac{\mu}{2}}}{k^{\frac{\mu-1}{2}} e^{k \mu} \Omega_{1}^{\frac{\mu}{2}}} \sum_{i_{1}=1}^{\infty}\left(\mu \sqrt{\frac{k(k+1)}{\Omega_{1}}}\right)^{2 i_{1}+\mu-1} . \\
& \cdot \frac{1}{i_{1} ! \Gamma\left(i_{1}+\mu\right)} x_{1}^{2 i_{1}+2 \mu-1} e^{-\frac{\mu(k+1)}{\Omega_{1}} y_{1}^{2}} \frac{\mu(k+1)^{\frac{\mu+1}{2}}}{k^{\frac{\mu-1}{2}} e^{k \mu} \Omega_{1}^{\frac{\mu+1}{2}}} . \\
& \cdot \sum_{i_{2}=1}^{\infty}\left(\mu \sqrt{\frac{k(k+1)}{\Omega_{1}}}\right)^{2 i_{2}+\mu-1}\left(\frac{\Omega_{1}}{\mu(k+1)}\right)^{i_{2}+\mu} . \\
& \cdot \frac{1}{i_{2} ! \Gamma\left(i_{2}+\mu\right)} \gamma\left(i_{2}+\mu, \frac{\mu(k+1)}{\Omega_{1}} y_{1}^{2}\right) .
\end{aligned}
$$

The average level crossing rate of the second micro-diversity SC output signal is:

$$
\begin{aligned}
& N_{x_{2}}=\frac{2 \sqrt{2 \pi} f_{m} \mu^{\frac{1}{2}}(k+1)^{\frac{\mu}{2}}}{k^{\frac{\mu-1}{2}} e^{k \mu} \Omega_{2}^{\frac{\mu}{2}}} \sum_{i_{1}=1}^{\infty}\left(\mu \sqrt{\frac{k(k+1)}{\Omega_{1}}}\right)^{2 i_{1}+\mu-1} . \\
& \cdot \frac{1}{i_{1} ! \Gamma\left(i_{1}+\mu\right)} x_{2}^{2 i_{1}+2 \mu-1} e^{-\frac{\mu(k+1)}{\Omega_{2}} y_{2}{ }^{2}} \frac{\mu(k+1)^{\frac{\mu+1}{2}}}{k^{\frac{\mu-1}{2}} e^{k \mu} \Omega_{2}^{\frac{\mu+1}{2}}} . \\
& \cdot \sum_{i_{2}=1}^{\infty}\left(\mu \sqrt{\frac{k(k+1)}{\Omega_{2}}}\right)^{2 i_{2}+\mu-1}\left(\frac{\Omega_{2}}{\mu(k+1)}\right)^{i_{2}+\mu} \cdot \\
& \cdot \frac{1}{i_{2} ! \Gamma\left(i_{2}+\mu\right)} \gamma\left(i_{2}+\mu \frac{\mu(k+1)}{\Omega_{2}} y_{2}{ }^{2}\right) .
\end{aligned}
$$

LCR of MaD SSC output signal envelope is equal to LCR of the first MiD SC output signal envelope when the first MiD SC provides signal to mobile user and the total power at its inputs is higher than predetermined threshold or when the second MiD SC receiver provides signal to the mobile user and the total power at its inputs is lower than the threshold. On the other hand, LCR of MaD SSC output signal envelope is equal to LCR of the second MiD SC output signal envelope when the second MiD SC provides the signal to mobile user and the total power at its inputs is higher than the predetermined threshold or when the first MiD SC provides the mobile user and the total power at its input is lower than the predetermined threshold.

Accordingly, the LCR of MaD SSC output signal envelope is: 


$$
\begin{aligned}
\mathrm{N}_{\mathrm{x}} & =\frac{1}{2} \int_{\Omega_{\mathrm{T}}}^{\infty} \mathrm{d} \Omega_{1} \int_{0}^{\infty} \mathrm{N}_{\mathrm{y} \mid \Omega_{1}} \mathrm{p}_{\Omega_{1} \Omega_{2}}\left(\Omega_{1} \Omega_{2}\right) \mathrm{d} \Omega_{2}+ \\
& +\frac{1}{2} \int_{0}^{\Omega_{\mathrm{T}}} \mathrm{d} \Omega_{1} \int_{0}^{\infty} \mathrm{N}_{\mathrm{y} \mid \Omega_{2}} \mathrm{p}_{\Omega_{1} \Omega_{2}}\left(\Omega_{1} \Omega_{2}\right) \mathrm{d} \Omega_{2}+ \\
& +\frac{1}{2} \int_{\Omega_{T}}^{\infty} d \Omega_{2} \int_{0}^{\infty} N_{y \mid \Omega_{2}} p_{\Omega_{1} \Omega_{2}}\left(\Omega_{1} \Omega_{2}\right) d \Omega_{1}+ \\
& +\frac{1}{2} \int_{0}^{\Omega_{T}} d \Omega_{2} \int_{0}^{\infty} N_{y \mid \Omega_{1}} p_{\Omega_{1} \Omega_{2}}\left(\Omega_{1} \Omega_{2}\right) d \Omega_{1}= \\
= & \int_{\Omega_{T}}^{\infty} d \Omega_{1} \int_{0}^{\infty} N_{y \mid \Omega_{1}} p_{\Omega_{1} \Omega_{2}}\left(\Omega_{1} \Omega_{2}\right) d \Omega_{2}+ \\
& +\int_{0}^{\Omega_{\mathrm{T}}} \mathrm{d} \Omega_{2} \int_{0}^{\infty} \mathrm{N}_{\mathrm{y} \mid \Omega_{2}} \mathrm{p}_{\Omega_{1} \Omega_{2}}\left(\Omega_{1} \Omega_{2}\right) \mathrm{d} \Omega_{1}=\mathrm{I}_{1}+\mathrm{I}_{2} .
\end{aligned}
$$

where $N_{y \mid \Omega_{1}}$ and $\mathrm{N}_{\mathrm{y} \mid \Omega_{2}}$ LCR at the first and second input of SSC obtained in (9) and (10), respectively and $p_{\Omega_{1} \Omega_{2}} \Omega_{1} \Omega_{2}$ is Gamma distribution already given in

The LCR of MaD system with MaD SSC receiver and two MiD SC branches can be obtained by solving the following integrals (Gradshteyn \& Ryzhik, 2000):

$$
\begin{aligned}
& I_{1}=\int_{\Omega_{T}}^{\infty} d \Omega_{1} \int_{0}^{\infty} d \Omega_{2} N_{y_{1}}\left(y \mid \Omega_{1}\right) p_{\Omega_{1} \Omega_{2}}\left(\Omega_{1} \Omega_{2}\right)= \\
& =\frac{2 \sqrt{2 \pi} f_{m} \mu^{\frac{3}{2}}(k+1)^{\mu+\frac{1}{2}}}{k^{\mu-1} e^{2 k \mu}} \sum_{j_{1}=0}^{\infty} \mu \sqrt{k(k+1)}{ }^{2 j_{1}+\mu-1} \\
& \frac{1}{j_{1} ! \Gamma\left(j_{1}+\mu\right)} \mathrm{y}^{2 j_{1}+2 \mu-1} \sum_{j_{2}=0}^{\infty} \mu \sqrt{k(k+1)}^{2 j_{2}+\mu-1} \text {. } \\
& \frac{1}{j_{2} ! \Gamma\left(j_{2}+\mu\right)}\left(\frac{1}{\mu(k+1)}\right)^{j_{2}+\mu} \frac{1}{\Gamma(c)\left(1-\rho^{2}\right) \rho^{c-1} \Omega_{0}^{c+1}} \cdot \\
& \cdot \sum_{j_{3}=0}^{\infty}\left(\frac{\rho}{\Omega_{0}\left(1-\rho^{2}\right)}\right)^{2 j_{3}+c-1} \frac{1}{j_{3} ! \Gamma\left(j_{3}+c\right)}\left(\Omega_{0}\left(1-\rho^{2}\right)\right)^{i_{3}+c} . \\
& \cdot \Gamma\left(i_{3}+c\right) \int_{\Omega_{T}}^{\infty} \Omega_{1}^{j_{3}+c-\frac{1}{2}-\mu-j_{1}} \gamma\left(j_{2}+\mu, \frac{\mu(k+1)}{\Omega_{1}} y^{2}\right) . \\
& \cdot e^{-\frac{\mu(k+1)}{\Omega_{1}} y^{2}-\frac{\Omega_{1}}{\Omega_{0}\left(1-\rho^{2}\right)}} d \Omega_{1} \text {. }
\end{aligned}
$$

Integral $I_{2}$ is (Gradshteyn \& Ryzhik, 2000):

$$
\begin{aligned}
& I_{2}=\int_{0}^{\Omega_{r}} d \Omega_{2} \int_{0}^{\infty} d \Omega_{1} N_{x_{1}}\left(x \mid \Omega_{1}\right) p_{\Omega_{1} \Omega_{2}}\left(\Omega_{1} \Omega_{2}\right)= \\
& =\frac{2 \sqrt{2 \pi} f_{m} \mu^{\frac{3}{2}}(k+1)^{\mu+\frac{1}{2}}}{k^{\mu-1} e^{2 k \mu}} \sum_{j_{1}=0}^{\infty} \mu \sqrt{k(k+1)}^{2 j_{1}+\mu-1} .
\end{aligned}
$$

$$
\begin{aligned}
& \cdot \frac{1}{j_{1} ! \Gamma\left(j_{1}+\mu\right)} \mathrm{y}^{2 j_{1}+2 \mu-1} \sum_{j_{2}=0}^{\infty} \mu \sqrt{k(k+1)}{ }^{2 j_{2}+\mu-1} . \\
& \cdot \frac{1}{j_{2} ! \Gamma\left(j_{2}+\mu\right)}\left(\frac{1}{\mu(k+1)}\right)^{j_{2}+\mu} \frac{1}{\Gamma(c)\left(1-\rho^{2}\right) \rho^{c-1} \Omega_{0}^{c+1}} \cdot \\
& \cdot \sum_{j_{3}=0}^{\infty}\left(\frac{\rho}{\Omega_{0}\left(1-\rho^{2}\right)}\right)^{2 j_{3}+c-1} \frac{1}{j_{3} ! \Gamma\left(j_{3}+c\right)}\left(\Omega_{0}\left(1-\rho^{2}\right)\right)^{i_{3}+c} \\
& \cdot \gamma\left(i_{3}+c, \frac{\Omega_{T}}{\Omega_{0}\left(1-\rho^{2}\right)}\right) \int_{\Omega_{T}}^{\infty} \Omega_{1}^{j_{3}+c-\frac{1}{2}-\mu-j_{1}} . \\
& \cdot \gamma\left(j_{2}+\mu, \frac{\mu(k+1)}{\Omega_{1}} y^{2}\right) e^{-\frac{\mu(k+1)}{\Omega_{1}} y^{2}-\frac{\Omega_{1}}{\Omega_{0}\left(1-\rho^{2}\right)} d \Omega_{1} .}
\end{aligned}
$$

\section{NUMERICAL RESULTS}

Average level crossing rate (LCR) is important second order performance measure of wireless communication system, which determines the number of signal threshold crossings in positive going direction.

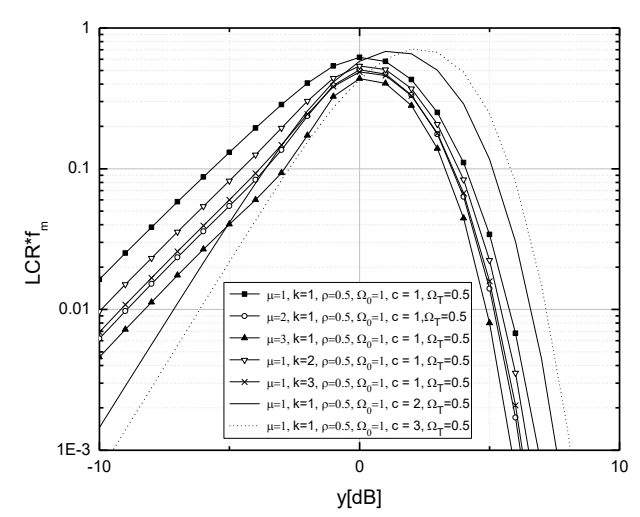

Fig. 2. Normalized LCR of MaD SSC receiver for several values of parameter mu, kappa and $\mathrm{c}$ and constant values of $\rho, \Omega_{0}$, and $\Omega_{T}$.

Figure 2. presents normalized LCR of MaD SSC system for various values of fading severity parameters $\mu$, Rice factor $k$ shadowing severity $c$, and constant values of correlation parameter $\rho$, average power $\Omega_{0}$ and threshold value $\Omega_{T}$. It is evident that LCR increases for lower values of $y$, reaches its maximum, and then decreases for higher values of $y$. It can been seen that by increasing parameter $\mu$ and $k$, it comes to the improvement of the performances of MaD SSC system, since LCR decreases. For lower values of $y$, LCR decreases as parameter $\mathrm{c}$ increases while for higher values of $y$, LCR increases as parameter $c$ increases. Moreover, parameter $c$ has greater impact on LCR then parameter $\mu$ and $k$. 


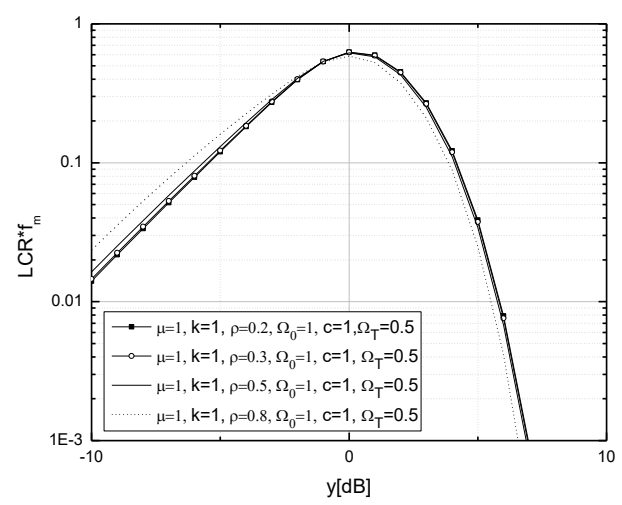

Fig. 3. Normalized LCR of MaD SSC receiver for constant values of mu, kappa, c, $\Omega_{0}$ and $\Omega_{T}$ and several values of $\rho$.

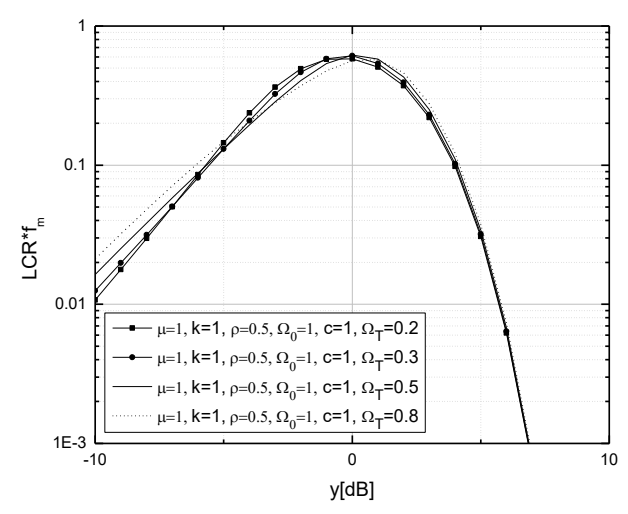

Fig. 4. Normalized LCR of MaD SSC receiver for constant values of mu, kappa, $c, \Omega_{0}$ and $\rho$ and several values of $\Omega_{T}$.

Fig. 3. shows normalized average level crossing rate versus $\mathrm{y}$ for various values of $\rho$ and constant values of $\mu, k, c, \Omega_{0}$ and $\Omega_{T}$. Since there is no correlation for $\rho=0$, the impact of $\rho$ on LCR can be noticed for higher values of $\rho$.

Fig. 4. shows normalized average level crossing rate versus y for various values of $\Omega_{T}$ and constant values of $\mu, k, c, \Omega_{0}$ and $\rho$.

\section{CONCLUSION}

In this paper, MaD technique with $\mathrm{MaD}$ switch and stay combining reception and two MiD selection combiners in correlated Gamma shadowed Kappa-Mu multipath fading channel is considered. MaD system with MaD SSC receiver is proposed, since it has lower complexity implementation then other diversity techniques. LCR of SSC MaD receiver output signal envelope in the form the sum of one-folded integrals is obtained and graphically presented in relation to different system model parameters.

\section{REFERENCES}

Basnayaka, D.A., Smith, P.J., \& Martin, P.A. 2013. The effect of macrodiversity on the performance of maximal ratio combining in flat Rayleigh fading. IEEE Transactions on Communications, 61(4), pp. 1384-1392.

Djosic, D., Stefanovic, D., \& Stefanovic, C. 2016. Level Crossing Rate of Macro-diversity System with Two Micro-diversity SC Receivers over Correlated Gamma Shadowed $\alpha-\mu \quad$ Multipath Fading Channels. IETE Journal of Research, 62(2), pp. 1-6.

Gradshteyn, I.S., \& Ryzhik, I.M. 2000. Table of Integrals, Series, and Products, 6th ed..New York: Academic.

Milic, D., Djosic, D., Stefanovic, C., Panic, S., \& Stefanovic, S.M. 2016. Second order statistics of the $\mathrm{SC}$ receiver over Rician fading channels in the presence of multiple Nakagami-m interferers. International Journal of Numerical Modelling: Electronic Networks, Devices and Fields, 29(2), pp. 222-229.

Mukherjee, S., \& Avidor, D. 2003. Effect of microdiversity and correlated macrodiversity on outages in a cellular system. IEEE Trans. on Wireless Technol, 2(1), pp. 50-59.

Panić, S., Stefanovic, D., Petrović, I., Stefanović, M., Anastasov, J., \& Krstic, D. 2011. Second order statistics of selection macro-diversity system operating over Gamma shadowed $k-\mu$ fading channels. EURASIP Journal on Wireless Communications and Networking, 2011. Oct.

Panic, S., Stefanovic, M., Anastasov, J., \& Spalevic, P. 2013. Fading and Interference Mitigation in Wireless Communications.New York: CRC Press.

Paris, J.F. 2014. Statistical Characterization of $\mathrm{k}-\mu$ Shadowed Fading. IEEE Transactions on Vehicular Technology, 63(2), pp. 518-526.

Shankar, P.M. 2009. Macrodiversity and microdiversity in correlated shadowed fading channels. IEEE Trans. onVehicular Technol, 2(58), pp. 727-732.

Stamenović, G., Panić, S., Rančić, D., Stefanović, C., \& Stefanović, M. 2014. Performance analysis of wireless communication system in general fading environment subjected to shadowing and interference. EURASIP Journal on Wireless Communications and Networking, 1, pp. 1-8.

Stefanovic, Č., Jakšić, B., Spalević, P., Panić, S., \& Trajčevski, Z. 2013. Performance analysis of selection combining over correlated Nakagami-m fading channels with constant correlation model for desired signal and cochannel interference. Radioengineering, 22(4), pp. 11761181. Dec. 
Stefanović, ¿̌. 2015. Macrodiversity system with macrodiversity SSC receiver and two microdiversity receivers in the presence of composite fading environment. . In: IEEE 23rd Telecommunications forum - TELFOR 2015, Proceedings of papers, 2015-11-24, Belgrade, Serbia. , pp. 321-324.

Stefanović, C., Petkovič, M., Nikolić, B., \& Đorđević, G. 2015. Effect of phase noise on error performance of DE-QPSK receiver over $\mathrm{k}-\mu$ fading channel. . In: IEEE 23rd Telecommunications forum - TELFOR 2015, Proceedings of papers, 2015-11-24, Belgrade, Serbia. , pp. 301-304.

Stefanović, D., Stefanović, Č., Rančić, D., \& Stefanović, M.. Outage probability of macrodiversity system in the presence of Gamma long term fading Rayleigh short term fading and Nakagami-m CCI. . In: Proc. IEEE Eight International Conference on Ubiquitous and Future Networks (ICUFN) 2016, Proceedings of papers, 2016-07-05, Vienna, Austria. , pp. 259-263.

Stüber, G.L. 1996. Principles of Mobile Communications. Massachusetts, USA: Kluwer Academic Publishers.

Xekalaki, E., Panaretos, J., \& Psarakis, S. 2003. A predictive model evaluation and selection approach- the correlated gamma ratio distribution. In $\mathbf{J}$. Panaretos Ed., Stochastic musings: Perspectives from the pioneers of the late 20th century.USA: Psychology Press., pp. 188-202.

Yue, S., Ouarda, T.B.M.J., \& Bobee, B. 2001. A review ofbivariate gamma distributions for hydrological application. J. Hydrol, 246, pp. 1-18.

Yacoub, M.D. 2007. The k- $\mu$ distribution and the $\eta-\mu$ distribution. IEEE Antennas and Propagation Magazine, 49(1), pp. doi:10.1109/MAP.2007.370983.

Zhang, B., Zhong, Z., Ai, B., \& He, R.. Impact of Shadowing Correlation on Microdiversity and Marcodiversity of Cellular System in High-speed Railway Environments. . In: Proc. Progress In Electromagnetics Research Symposium, 2014-08-25, Guangzhou, China. , pp. 2159-2163

Zhao, J., Fan, P., Beaulieu, N.C., \& Lei, X.. Switching rates of selection diversity and switch-and stay diversity on mixed high-speed train channels. . In: Proc. IEEE International Workshop on High Mobility Wireless Communications, 2015-10-21, Xi'an, China. , pp. 51-55.

*E-mail: danijel.djosic@pr.ac.rs 